\title{
Hyperlactataemia with acute kidney injury following community assault: cause or effect?
}

\author{
David Lee Skinner, ${ }^{1}$ Carolyn Lewis, ${ }^{2}$ Kim de Vasconcellos, ${ }^{1}$ John Bruce, ${ }^{3}$ Grant Laing, ${ }^{3}$ Damian Clarke, ${ }^{3}$ David Muckart ${ }^{3}$ \\ ${ }^{1}$ Perioperative Research Group: Department of Anaesthetics and Critical Care, University of KwaZulu-Natal \\ ${ }^{2}$ Division of Emergency Medicine, University of Witwatersrand, Johannesburg, South Africa \\ ${ }^{3}$ Department of Surgery, University of KwaZulu-Natal
}

Corresponding author: David Lee Skinner (drdavidskinner@gmail.com)

\begin{abstract}
Background: Crush injury is a common presenting clinical problem in South African trauma patients, causing acute kidney injury (AKI). It has been theorised previously that the AKI was not due to an anaerobic phenomenon. A previous local study noted the presence of a mild hyperlactataemia among patients with crush syndrome, but the significance and causes of this was not fully explored. This study aimed to examine the incidence of hyperlactataemia in patients with crush syndrome presenting to a busy emergency department (ED) in rural South Africa.

Methods: The study was conducted at Edendale Hospital in KwaZulu-Natal province in South Africa from 1 June 2016 to 31 December 2017. All patients from the ED who had sustained a crush injury secondary to a mob assault were included in the study. Patients with GCS on arrival of $\leq 13$ or polytrauma were excluded from analysis. The primary outcome of interest was the presence of hyperlactataemia $(\geq 2.0 \mathrm{mmol} / \mathrm{L})$ on presentation. The Kidney Disease Improving Global Outcomes (KDIGO) criteria were used to diagnose and stage AKI as a secondary outcome.

Results: A total of 84 patients were eligible for analysis. Sixty-nine (82\%) patients presented with hyperlactataemia. The median serum lactate was $4.9 \mathrm{mmol} / \mathrm{L}$ (IQR $2.3-7.2 \mathrm{mmol} / \mathrm{L}$ ). Fifteen $(18 \%)$ patients were diagnosed with AKI on presentation according to serum creatinine. Ten patients were diagnosed as Stage 1, three were Stage 2 and two Stage 3 AKI respectively. There was no difference in the incidence of AKI in patients with or without hyperlactataemia $(p=0.428)$. Time from injury to presentation was a median 365 minutes (IQR 180-750 minutes). Six (7\%) patients were admitted to high care unit and nine (11\%) were admitted to the intensive care unit (ICU). No patients died within 48 hours of admission. Two patients received renal replacement therapy during the first 48 hours of admission to hospital.

Conclusion: Hyperlactataemia is a common feature of patients presenting to the ED following crush syndrome secondary to beatings received during interpersonal violence. The origin of this hyperlactataemia is currently unknown. Further research needs to be conducted into the origin of the hyperlactataemia and its clinical significance. In this cohort, the utilisation of RRT was low but the incidence of AKI was high and developed rapidly following the injury. The utilisation of RRT also needs to be further studied in larger patient populations in South Africa to make local clinical recommendations for use.

Keywords: Acute Kidney Injury, Crush Syndrome, Rhabdomyolysis, Trauma
\end{abstract}

S Afr J Surg 2019;57(2)

http://dx.doi.org/10.17159/2078-5151/2019/v57n2a2779

\section{Introduction}

Crush injury was first described in victims of urban bombing attacks during the London Blitz in $1940 .{ }^{1}$ These injuries are well recognised today in victims of entrapments following both natural disasters and civilian bombings. ${ }^{2,3}$ These injuries are ischaemic in nature and reflect the end result of prolonged hypoperfusion of a trapped limb. Once the patient is extracted and the limb freed, uncontrolled reperfusion of the limb leads to a massive systemic response which may be acutely fatal or precipitate acute kidney injury (AKI). The main mechanism of this AKI is believed to be secondary to the deposition of myoglobin into the renal tubules.

In South Africa, a variant on the classic crush injury is commonly seen following so called mob assaults following community vigilante action against victims accused of crimes. These patients sustain a beating from the community using punches and kicks as well as a variety of blunt objects. The classic South African whip known as the sjambok has been used frequently in these attacks. These patients are common in South African emergency departments (EDs). ${ }^{4-6}$ AKI that can occur as a consequence of this injury can be difficult to manage and may result in the need for renal replacement therapy (RRT).

Although much has been written on crush syndromes following patients freed from entrapment ${ }^{7,8}$ less has been documented about crush syndrome secondary to community 
assault where limb entrapment is not a major feature. It has been suggested that the crush syndrome from beating with sticks and sjamboks is an aerobic phenomenon, dominated by the presence of myoglobinaemia and without the toxic effects of anaerobic metabolites which characterise the crush syndrome with reperfusion of a limb with a compromised vascular supply. ${ }^{5,9,10}$ A previous study noted the presence of a mild hyperlactataemia among patients with crush syndrome, but the significance of this was not fully explored. ${ }^{5}$ This study aimed to examine the incidence of hyperlactataemia in patients with crush syndrome presenting to a busy ED in rural South Africa following beatings. The incidence of AKI within 48 hours of presentation to ED was examined as a secondary outcome.

\section{Methods}

\section{Setting}

The study was conducted at Edendale Hospital, a rural regional level hospital set on the outskirts of Pietermaritzburg, the capital city of KwaZulu-Natal province in South Africa. It borders a large informal settlement and receives a high burden of trauma referrals from the surrounding areas (approximately $1200 /$ month based on previous audits). A database maintained by the Pietermaritzburg Metropolitan Trauma Service (PMTS) was interrogated for the time period from 1 June 2016 to 31 December 2017. Ethics approval to maintain the registry has been obtained from the Biomedical Research Ethics Committee (BCA221/13 BREC) of the University of Kwa Zulu-Natal and from the Research Unit of the Department of Health. ${ }^{11}$

\section{Definitions and outcomes}

All patients from the ED and subsequently referred to the PMTS for admission who had sustained a crush injury secondary to a mob or community assault were included in the study. For the purposes of this study, crush syndrome was defined as injuries sustained via interpersonal violence and beating with a blunt object such as sticks, whips or sjamboks. Patients included in the study had clinical evidence of skin bruising from blunt trauma. Urine dipstick tests were performed on all patients and were positive for haemoglobin pigment. Patients with significant head injuries defined as a GCS on arrival of $\leq 13$ were also excluded due to the difficulty in interpreting elevated serum lactate in traumatic brain injury (TBI). Polytrauma patients were also excluded from analysis, as they may have other reasons for hyperlactataemia, such as compromised limb or organ vascular supply. Polytrauma was defined as a patient with significant injuries in two or more anatomical regions.

Presentation biochemistry, demographic data and data up to 48 hours following admission to the ED were used for analysis. No further follow up after 48 hours was performed. The primary outcome of interest was the presence of hyperlactataemia on presentation defined as a serum lactate $\geq 2.0 \mathrm{mmol} / \mathrm{L}$. The presence of AKI on admission or developing within 48 hours following admission was a secondary outcome. Admission to the intensive care unit (ICU) or the high care unit (HCU) was also examined as secondary outcomes. The criteria for admission to these units included the need for more advanced monitoring than offered in the general ward, or the need for organ support in the form of ventilation, inotropic support or RRT offered in the ICU.

Treatment of these patients followed a protocol using venous serum bicarbonate for risk stratification, administration of an intravenous balanced salt solution and targeting a urine output of $1-2 \mathrm{ml} / \mathrm{kg} / \mathrm{hr}$ with clinical assessment of fluid balance and referral as required for RRT. (See Annexure A for detailed protocol.)

AKI was defined according to the current Kidney Disease Improving Global Outcomes (KDIGO) diagnostic criteria using either urine output or serum creatinine levels. ${ }^{12}$ If the baseline serum creatinine was unknown, it was calculated according to the Modification of Disease in Renal Diet (MDRD) equation, as per the KDIGO recommendations. ${ }^{12}$

\section{Statistical analysis}

Means and standard deviations are reported for normally distributed data; median and inter-quartile range for data not normally distributed. The $\mathrm{x} 2$ test and Fisher's exact test were used for categorical data, and independent samples t-test, Kruskall-Wallis, or Mann-Whitney U test for continuous data where appropriate. All p-values are reported to three decimal places and statistical significance was defined as a two-sided $\mathrm{p}$-value $<0.05$. All data analyses were performed using SPSS 24.0 for Mac (SPSS, IBM, Chicago, IL).

\section{Results}

A total of 84 patients were eligible for analysis. The majority were young males (Table 1). Sixty-nine (82\%) patients presented with hyperlactataemia. Three patients had missing admission serum lactate levels.

\begin{tabular}{|c|c|}
\hline Age (years, median, IQR) & $25(21-30)$ \\
\hline Male gender (n, \%) & $82(98)$ \\
\hline SBP (mmHg, median, IQR) & $118(102-131.5)$ \\
\hline DBP (mmHg, median, IQR) & $76(67.5-88.5)$ \\
\hline Pulse (bpm, median, IQR) & $92(79-103.5)$ \\
\hline GCS (median, IQR) & $15(15-15)$ \\
\hline Haematuria (dipstick) (n, \%) & $80(95 \%)$ \\
\hline $\begin{array}{l}\text { Time from injury to presentation } \\
\text { (minutes, median, IQR) }\end{array}$ & $365(180-750)$ \\
\hline \multicolumn{2}{|c|}{$\begin{array}{l}\mathrm{SBP}=\text { systolic blood pressure. } \mathrm{DBP}=\text { diastolic blood pressure } \\
\text { GCS = Glasgow Coma Scale. }\end{array}$} \\
\hline
\end{tabular}

Forty-nine $(58 \%)$ patients presented with acidaemia ( $\mathrm{pH}$ $<7.35$ ). The presentation biochemistry for patients with and without hyperlactataemia are presented in Table 2 . There were significant differences in the presentation $\mathrm{pH}$ and base excess, 
Table 2: Presentation biochemistry of patients with and without hyperlactataemia $(n=81 *)$

\begin{tabular}{lcccc}
\hline $\begin{array}{l}\text { All data presented as } \\
\text { median }(\mathbf{I Q R}) \text { or } \mathbf{n}(\mathbf{\%})\end{array}$ & Whole Cohort $(\mathbf{n = 8 1 )}$ & $\begin{array}{c}\text { Hyperlactataemia } \\
(\mathbf{n = 6 7 )}\end{array}$ & $\begin{array}{c}\text { No hyperlactataemia } \\
(\mathbf{n = 1 4})\end{array}$ & P Value \\
\hline $\mathrm{pH}$ & $7.312(7.213-7.370)$ & $7.290(7.200-7.350)$ & $7.400(7.370-7.440)$ & $<0.001$ \\
Base excess $(\mathrm{mmol} / \mathrm{L})$ & $-5.4(-9.3--2.6)$ & $-5.8(-10.3--3.6)$ & $-1.8(-4.2--0.9)$ & 0.001 \\
Lactate $(\mathrm{mmol} / \mathrm{L})$ & $4.9(2.3-7.2)$ & $5.5(3.7-7.7)$ & $1.1(0.9-1.6)$ & $<0.001$ \\
Sodium $(\mathrm{mmol} / \mathrm{L})$ & $140(138-143)$ & $140(138-143)$ & $140(136-141)$ & 0.338 \\
Potassium $(\mathrm{mmol} / \mathrm{L})$ & $4.1(3.8-4.8)$ & $4.1(3.7-4.8)$ & $4.4(3.9-5.4)$ & 0.337 \\
Chloride $(\mathrm{mmol} / \mathrm{L})$ & $104(102-107)$ & $104(102-106)$ & $105(102-108)$ & 0.233 \\
HCO3 $(\mathrm{mmol} / \mathrm{L})$ & $20(17-22)$ & $20(17-22)$ & $23(17-26)$ & 0.059 \\
Urea $(\mathrm{mmol} / \mathrm{L})$ & $5.8(4.1-7.8)$ & $5.8(4.1-7.3)$ & $6.4(4.4-11.2)$ & 0.281 \\
Creatinine $(\mu \mathrm{mol} / \mathrm{L})$ & $99(90-137)$ & $99(80-128)$ & $101(72-215)$ & 0.554 \\
Hb $(\mathrm{g} / \mathrm{dL})$ & $12.9(11.2-14.4)$ & $12.9(11.3-14.5)$ & $12.1(8.4-14.1)$ & 0.189 \\
Platelets $(x 10$ /L) & $225(178-272)$ & $232(203-275)$ & $148(126-207)$ & 0.001 \\
AKI $(\mathrm{n}, \%)$ & $14(17)$ & $11(16)$ & $3(21)$ & 0.629 \\
*Three patients with missing lactate data. & & &
\end{tabular}

and no difference in the presentation serum bicarbonate between patients with or without hyperlactataemia. There was no statistical difference in the median systolic blood pressure $(\mathrm{p}=0.224)$ or median diastolic blood pressure $(\mathrm{p}=0.919)$ in patients with or without hyperlactataemia.

The differences between the patients with and without AKI are presented in Table 3. The patients with AKI had significantly lower median $\mathrm{pH}$, lower base excess and higher serum lactate. They also had a higher median serum potassium and a lower median haemoglobin.

No patients had serum creatinine kinase levels performed. Fifteen $(18 \%)$ patients were diagnosed with AKI on presentation according to serum creatinine. Only 2 of those patients were also diagnosed with AKI according to urine output criteria within the 48 -hour time period. Ten patients were diagnosed as Stage 1, three were Stage 2 and two Stage 3 all according to serum creatinine values. There was no difference in the incidence of AKI in patients with or without hyperlactataemia $(p=0.428)$.
Although there was a slightly longer delay in patients with AKI presenting to the ED, there was no statistically significant difference noted (median 360 minutes; IQR 180-720 vs 495; $320-915 \mathrm{p}=0.143)$. There was a statistically shorter time to presentation in patients with hyperlactataemia (median 330; IQR160-550 vs 865; IQR 328-928).

Eighty-three $(99 \%)$ patients received either Modified Ringer's Lactate or Balsol ${ }^{\circledR}$ as resuscitation fluid initially. Five $(6 \%)$ patients also received $0.9 \%$ saline solution. No patients were given synthetic colloids or blood products as resuscitation fluids. The volume of oral or intravenous fluids administered was not reliably recorded for reporting.

Two (2\%) patients received a non-steroidal antiinflammatory drug on admission. No patients received intravenous (IV) mannitol, furosemide, sodium bicarbonate or an aminoglycoside in the first 48 hours following presentation, as per the local protocol for resuscitation of these patients.

Nine $(11 \%)$ patients were discharged within 24 hours, and $30(36 \%)$ were discharged within 48 hours. Six $(7 \%)$ patients

Table 3: Presentation biochemistry of patients with and without AKI with crush syndrome (n=84)

\begin{tabular}{lcccc}
\hline All data presented as median $(\mathbf{I Q R})$ & Whole Cohort $(\mathbf{n = 8 4})$ & No AKI $(\mathbf{n}=\mathbf{6 9})$ & AKI (n=15) & P Value \\
\hline $\mathrm{pH}$ & $7.314(7.220-7.370)$ & $7.320(7.260-7.3700$ & $7.210(7.060-7.320)$ & 0.025 \\
Base excess $(\mathrm{mmol} / \mathrm{L})$ & $-5.4(-9.0--2.6)$ & $-5.2(-7.3--2.6)$ & $-10.2(-14.2--4.6)$ & 0.025 \\
Lactate $(\mathrm{mmol} / \mathrm{L})$ & $4.9(2.3-7.2)$ & $4.2(2.9-5.7)$ & $7.2(4.0-12.0)$ & 0.043 \\
Sodium $(\mathrm{mmol} / \mathrm{L})$ & $140(138-142)$ & $140(138-142)$ & $138(137-142)$ & 0.271 \\
Potassium $(\mathrm{mmol} / \mathrm{L})$ & $4.2(3.8-4.8)$ & $4.1(3.7-4.6)$ & $5.1(4.5-5.9)$ & 0.002 \\
Chloride $(\mathrm{mmol} / \mathrm{L})$ & $104(102-107)$ & $104(102-107)$ & $104(102-106)$ & 0.942 \\
$\mathrm{HCO}_{3}^{-}(\mathrm{mmol} / \mathrm{L})$ & $20(17-22)$ & $21(18-23)$ & $17(15-20)$ & 0.007 \\
Urea $(\mathrm{mmol} / \mathrm{L})$ & $5.8(5.3-7.9)$ & $4.9(3.8-6.3)$ & $10.1(8.5-16.9)$ & $<0.001$ \\
$\mathrm{Creatinine}(\mu \mathrm{mol} / \mathrm{L})$ & $99(79-139)$ & $93(73-110)$ & $200(184-343)$ & $<0.001$ \\
$\mathrm{Hb}(\mathrm{g} / \mathrm{dL})$ & $12.9(11.3-14.4)$ & $13.0(12.0-14.5)$ & $11.6(9.5-12.1)$ & 0.013 \\
Platelets $\left(\mathrm{x} 10^{9} / \mathrm{L}\right)$ & $224(183-273)$ & $225(203-272)$ & $195(159-247)$ & 0.207
\end{tabular}


were admitted to HCU and $9(11 \%)$ were admitted to the ICU. No patients died within 48 hours of admission. Two patients received RRT in the ICU during the first 48 hours of admission to hospital.

\section{Discussion}

Three decades ago, Muckart et al. reviewed crush syndrome in the South African context. ${ }^{10}$ They highlighted the fact that this syndrome was not due to entrapment and reperfusion, but rather due to a severe beating over the entire torso and limbs. They hypothesised that this was not an ischaemic phenomenon but rather the result of direct muscle disruption and the systemic release of myoglobin which is directly nephrotoxic. Most researchers in South Africa since have tended to support this hypothesis..$^{13,5,9}$

Our data present further hypotheses for the pathophysiology of crush syndrome in these patients. Contrary to what was expected, $82 \%$ of patients in our study had hyperlactataemia on presentation to the ED and those diagnosed with AKI on presentation were more likely to have higher serum lactate levels, although the presence of hyperlactataemia was not associated with the diagnosis of AKI. This study is the first to describe hyperlactataemia as a significant finding in this form of trauma.

There are a number of causes of hyperlactataemia that can be excluded as the incipient cause in our study cohort. Patients with significant head injuries were excluded from this study. The haemoglobin concentrations were similar in both groups with or without hyperlactataemia, and the median blood pressures did not represent a state of hypoperfusion. Furthermore, there was no difference in the presentation systolic or diastolic blood pressures between patients with and without hyperlactataemia, suggesting that hypotension was not the cause of hyperlactataemia.

A potential cause for the hyperlactataemia is that the areas of injury to the muscles that were damaged and crushed undergo a form of reperfusion and release lactate. As the amount of tissue reperfused increases, there is a greater potential for the release of nephrotoxins and sequestration of fluid in the damaged tissue's interstitum, resulting in AKI.

Enhanced glycolysis, which can occur in trauma and other inflammatory states such as septic shock, is another potential source for the hyperlactataemia. This occurs in the presence of normal aerobic metabolism. ${ }^{14,15}$

Damage associated molecular patterns (DAMPs) such as high mobility group box protein 1 (HMGBP-1) are known to be released during tissue damage in trauma. ${ }^{16}$ The kidneys filter 120-150 $\mathrm{ml}$ of plasma an hour, resulting in a significant exposure of both the glomeruli and tubules to these substances. Although not directly causative of inflammation, their activation of toll like receptors (TLRs) with subsequent inflammation of tissues is well described. ${ }^{17}$ These DAMPs may be associated with the presence of inflammation in the kidney, leading to AKI. The greater the number of circulating DAMPs, the greater the activation of the inflammation systems, which can lead to AKI.

A median 365-minute delay from injury represents a relatively short time from injury to presentation to hospital.
Despite this, a six-hour delay could result in sequestration of fluid in the damaged muscle, causing a relative hypovolaemia and subsequent hyperlactataemia. This can occur despite the normal haemodynamics on presentation, (pulse and blood pressure), as these markers are not sensitive enough to pick up smaller changes in intravascular volume shift.

Another potential theory is the presence of an intra-renal compartment syndrome, resulting in the decrease of renal blood flow and reducing glomerular filtration and peritubular vascular supply. The kidneys receive $20-25 \%$ of cardiac output and any reduction in perfusion results in significant ischaemia with tubular and glomerular damage. Swelling of the renal parenchyma may occur in response to inflammation, and this is worsened by inappropriate IV fluid therapy. The dense unyielding Gerota's fascia which surrounds the kidney would result in a rise in intra-renal compartment pressure. In a revisit to his seminal work on crush syndrome, Bywaters described the kidney at post-mortem as "overweight and oedematous, bulging out when the capsule was incised". ${ }^{18}$ This is similar to the behaviour of muscle that is released from a compartment syndrome. Renal ultrasound of patients with AKI often reveals enlarged and echogenic kidneys, with reduced intraparenchymal blood flow, making an intra-renal compartment syndrome a plausible hypothesis.

Lactate is removed from circulation by the liver predominantly, but the kidneys are responsible for some elimination. ${ }^{19}$ Reduction in clearance, in addition to an increase in metabolic demand by the injured muscle tissue may also explain the presence of the hyperlactataemia, despite the lack of obvious anaerobic tissue.

A unified hypothesis of the reasons patients with this form of crush syndrome would involve all potential causes as discussed. The muscle damage results in myoglobin release, there is sequestration of fluid within the damage muscle with subsequent hypovolaemia causing pre-renal dysfunction. This is then followed by the intrinsic renal damage caused by myoglobin and pro-inflammatory products released from the damaged tissue. Further swelling of the renal parenchyma from inappropriate fluid therapy and inflammation further reduces the intra-renal perfusion pressure, resulting in "multiple hits" to the kidney, with the end result being AKI requiring RRT.

Another surprise finding is the presence of AKI on presentation, despite a relatively short period of time between injury and presentation to the hospital. This might suggest that the renal dysfunction is far more rapid in onset than previously thought. KDIGO recommends examining a relatively small change in serum creatinine within 48 hours, but the patients in our study developed AKI within hours of their injury. The initial serum biochemistry was drawn prior to large volume IV fluid resuscitation, and within a reasonably short period of time following the trauma. All patients were referred from scene, with no inter-hospital transfers. This adds strength to the study as this is a better reflection of the biochemical disturbances these patients develop from their injuries without any fluid or pharmacological intervention prior to analysis.

NSAIDs are not indicated in the routine management of patients at risk for AKI, so our finding that 2 patients received NSAIDs whilst in the ED is unusual. As crush syndrome places 
patients at risk for AKI, all attempts to limit nephrotoxins should be made. Other, non-nephrotoxic analgesics should rather be used for pain control for this patient population.

Although only the type of fluid and not the quantity of fluid administered to the cohort was captured, the mainstay of therapy for patients with crush syndrome is IV fluid administration to induce a diuresis. In keeping with current evidence, no patients were given mannitol or sodium bicarbonate as part of their therapy. ${ }^{20}$ The use of furosemide is also not recommended in routine management of AKI and was not used in this patient cohort. ${ }^{12}$ Inappropriately small volumes of fluid could lead to under-resuscitation and worsening of potential AKI in patients with crush syndrome, but overloading patients with IV fluid in the presence of anuria can result in fluid overload, pulmonary oedema and hypoxia. As such, the careful monitoring of urine output and volumes of IV fluid administered are important in the management of these patients. ${ }^{7}$

There are a number of limitations to this study. The relatively small patient population limits external validity. Other hospitals in different areas of the country or hospitals in other countries experiencing this form of trauma may have different experiences of crush syndrome from severe beatings, with a variable time to presentation and severity of injury.

A significant number of patients present with crush syndrome and severe head injury, as that is the nature of the interpersonal violence seen by the ED. These patients were excluded to reduce the confounders to the reasons for hyperlactataemia, strengthening our findings as the crush syndrome being the main reason for the hyperlactataemia. This, however, limits the applicability of these findings to that patient cohort.

Although the type of fluid used was captured in the database, the volume of fluids administered to the patients was not, nor were intravascular volume assessment techniques recorded in the database. As the absolute volume of fluid administered is not predictive of the appropriateness of the fluid resuscitation, even capture and presentation of these data would not be clinically useful.

We do not have the data for prehospital vital signs and interventions that were offered to these patients. This would have influenced the presentation vital signs and biochemistry, depending on the resuscitation efforts that were received by the patients. As all patients were referred from the scene or self-presented, it is unlikely that large volumes of IV fluid were infused prior to presentation to the ED.

\section{Conclusion}

Hyperlactataemia is a common feature of patients presenting to the ED following crush syndrome secondary to beatings received during interpersonal violence. The origin of this hyperlactataemia is currently unknown and hypothesis generating. In this cohort, the utilisation of RRT was low but the incidence of AKI was high and developed rapidly following the injury. Further research needs to be conducted into the origin of the hyperlactataemia and its clinical significance. The utilisation of RRT also needs to be further studied in larger patient populations in South Africa to make local clinical recommendations for use.

\section{Acknowledgements}

The authors would like to thank Dr Ryan Keet for his assistance in the collection of data.

\section{REFERENCES}

1. Bywaters EG, Beall D. Crush Injuries with Impairment of Renal Function. Br Med J. 1941;1(4185):427-32.

2. Vanholder R, Sever MS, Erek E, Lameire N. Acute renal failure related to the crush syndrome: towards an era of seismonephrology? Nephrol Dial Transplant. 2000;15(10):1517-21. doi: $10.1093 / \mathrm{ndt} / 15.10 .1517$

3. Sever MS, Lameire N, Van Biesen W, Vanholder R. Disaster nephrology: a new concept for an old problem. Clin Kidney J. 2015;8(3):300-9. doi:10.1093/ckj/sfv024

4. Rosedale KJ, Wood D. Traumatic rhabdomyolysis (crush syndrome) in the rural setting. S Afr Med J. 2012;102(1):37-39.

5. Skinner DL, Laing GL, Bruce J, Biccard B, Muckart DJ. Validating the utilisation of venous bicarbonate as a predictor of acute kidney injury in crush syndrome from sjambok injuries. S Afr Med J. 2017;107(5):446-50. doi: 10.7196/SAMJ.2017. v107i5.12213

6. Bowley DMG, Buchan C, Khulu L, Boffard KD. Acute renal failure after punishment beatings. J R Soc Med. 2002;95(6):3001.

7. Gonzalez D. Crush syndrome. Crit Care Med. 2005; 33(1):S34-S41. doi: 10.1097/01.CCM.0000151065.13564.6F

8. Gibney RTN, Sever MS, Vanholder RC. Disaster nephrology: crush injury and beyond. Kidney Int. 2014;85(5):1049-57. doi: 10.1038/ki.2013.392

9. Smith WA, Hardcastle TC. A crushing experience: The spectrum and outcome of soft tissue injury and myonephropathic syndrome at an Urban South African University Hospital. African Journal of Emergency Medicine. 2011;1(1):17-24. doi: 10.1016/j.afjem.2011.04.002

10. Muckart DJ, Abdool-Carrim AT. Pigment-induced nephropathy after sjambok injuries. S Afr J Surg. 1991;29(1):21-4.

11. Laing GL, Bruce JL, Skinner DL, Allorto NL, Clarke DL, Aldous C. Development, implementation, and evaluation of a hybrid electronic medical record system specifically designed for a developing world surgical service. World J Surg. 2014;38(6):1388-97. doi: 10.1007/s00268-013-2438-2

12. Khwaja A. KDIGO clinical practice guidelines for acute kidney injury. Nephron Clin Pract. 2012;120(4):c179-c184. doi: $10.1159 / 000339789$

13. Skinner DL, Hardcastle TC, Rodseth RN, Muckart DJ. The incidence and outcomes of acute kidney injury amongst patients admitted to a level I trauma unit. Injury. 2014;45(1):259-64. doi: 10.1016/j.injury.2013.07.013

14. Levy B. Lactate and shock state: the metabolic view. Curr Opin Crit Care. 2006;12(4):315-21. doi: 10.1097/01. ccx.0000235208.77450.15

15. Kraut JA, Madias NE. Lactic acidosis. Ingelfinger JR, ed. N Engl J Med. 2014;371(24):2309-19. doi: 10.1056/NEJMra1309483

16. Land WG. The Role of Damage-Associated Molecular Patterns in Human Diseases: Part I - Promoting inflammation and immunity. Sultan Qaboos Univ Med J. 2015;15(1):e9-e21. doi: 10.1016/j.bcp.2014.01.036

17. Xiao W, Mindrinos MN, Seok J, et al. A genomic storm in critically injured humans. J Exp Med. 2011;208(13):2581-90. doi: 10.1084/jem.20111354

18. Bywaters EG. 50 years on: the crush syndrome. BMJ. 1990;301(6766):1412-5.

19. Bellomo R. Bench-to-bedside review: lactate and the kidney. Crit Care. 2014;18:3. 2002;6(4):322-6.

20. Brown CVR, Rhee P, Chan L, Evans K, Demetriades D, Velmahos GC. Preventing renal failure in patients with rhabdomyolysis: do bicarbonate and mannitol make a difference? Journal of Trauma and Acute Care Surgery. 2004;56(6):1191. Available from: http://dx.doi.org/10.1097/01. TA.0000130761.78627.10 